\title{
Fractal Minkowski Antenna Loaded with Hilbert Curve as Complementary Split Ring Resonator
}

\author{
Amer Basim Shaalan \\ Physics Department, College of Science, Al-Muthanna University, Iraq \\ *E-mail address: ame72r@gmail.com
}

Keywords: Left handed material; metamaterial; split ring resonator; fractal antenna; Hilbert curve

\begin{abstract}
Fractal Minkowski patch antenna is proposed. Classical complementary split ring resonator (CSRR) is slotted on the patch. For comparison, fractal Hilbert shape split ring resonator also slotted on the patch. These slots make the patch behaves as a left-handed material in certain frequency band. Minkowski fractal antenna has three resonance frequencies. The complementary split ring resonator and Hilbert ring affects the upper two frequencies and make the antenna resonate at lower frequencies. Lowering the resonance frequencies cause a reduction in antenna size. More reduction of antenna size is obtained when fractal Hilbert is used as complementary split ring resonator. The simulated results have been done by using (HFSS) software, which is based on finite element modeling. The measurement of fabricated antenna shows good agreement with simulation results.
\end{abstract}

\section{INTRODUCTION}

Few years ago, there is an interest among electromagnetic research groups in the study of Metamaterials as they provide electromagnetic properties not find in natural materials [1]. Simultaneously negative permeability and permittivity over certain frequency range are the most important properties of interest. Veselago while studying this type of materials in 1968 [2], he mentioned to some unique properties like negative refractive index and backward waves. Split ring resonator is one of the most known designs to get negative permeability. Several different shapes of split ring are published in literatures to achieve the requirement of negative permeability [3-5]. Classical microstrip patch antennas are the most popular antennas because of several advantages such as low profile, light weight and low cost of fabrication [6]. Antenna size is one of the most important parameters in many wireless applications. Although the microstrip antennas have low profile but it still not small enough in many wireless communication systems. Size reduction of antenna was investigated in many publications [7, 8]. Many techniques have been used to reduce antenna size, among these techniques is slotting the patch [9], using shorting posts [10] and using high permittivity dielectric substrate [11]. Fractal shapes are considered useful technique to get reduction in size. Many fractal antenna designs were published in recent years $[12,13]$. Left-handed materials are another technique for miniaturizing antenna size. Pendary was the first one who described negative permeability medium [14]. Later Smith implement first left handed material consist of periodic SRRs and long strips [15]. Recently, CSRR structure is used to reduce the microstrip antenna size [16].

In this paper, two techniques are used to reduce microstrip square patch antenna. Applying fractal Minkowski shape to the square patch antenna is the first step of miniaturization, and then the fractal patch is loaded with two different shapes of CSRR as a second step of miniaturization. Hilbert Curve is used as complementary split ring resonator to investigate the effect of the fractal shape in size reduction. The results are compared with the classical split ring resonator. More reduction in size is obtained when Hilbert shape is slotted on the patch. Good agreement of simulated results with measurement of fabricated models. 


\section{HILBERT RING DESIGN}

Hilbert Curve was first introduced by the German mathematician David Hilbert (1862-1943). This curve is called a space-filling curve, because it will fill the whole plane after multiple iterations as shown in Fig 1.
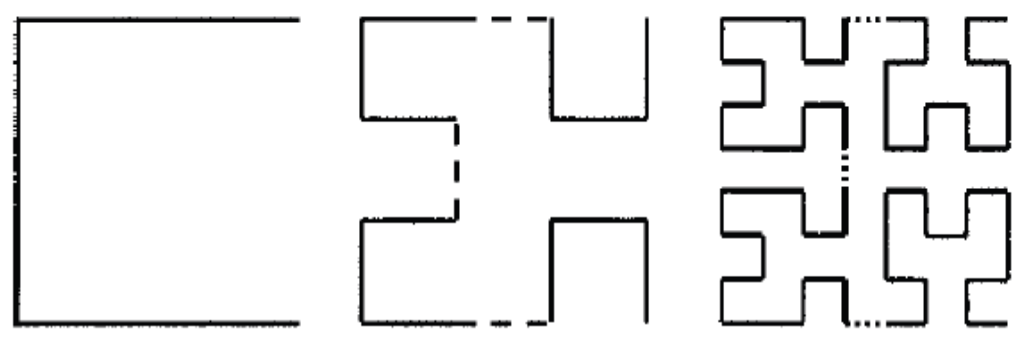

Fig. 1. Hilbert curve generation

In Hilbert curve generation process, each successive step consists of four copies of the previous step, connected with additional line segments (the dashed lines) [17].

\section{MINKOWSKI ANTENNA DESIGN}

The starting shape of this fractal is a square. Each of the four sides of the square is replaced by the generator. The generator is a straight segment divided into three segments, each segment is equal to one third (1/3) the length of the starting one. The middle one is removed from its place and connected with two other segments [18]. The generator is shown in Fig. 2.

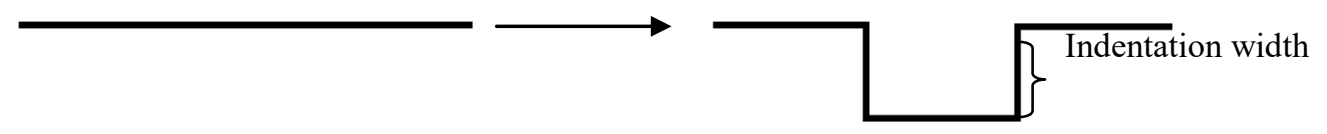

Fig. 2. The generator of Minkowiski Island

The two segments are tuned to adjust the overall perimeter of the fractal length. This tuning length is called the indentation width [19]. The whole shape of antenna is shown in Fig. 3. Patch antenna etched on a substrate of dimensions $65 \mathrm{~mm}$ x $65 \mathrm{~mm} \times 1.5 \mathrm{~mm}$. The substrate material is FR4 with relative permittivity $\left(\varepsilon_{\mathrm{r}}=4.4\right)$.
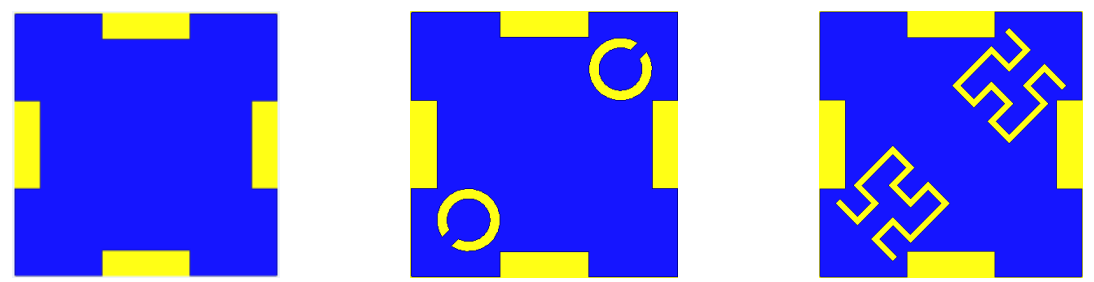

Fig. 3. Minkowiski Island antenna shape with ring and Hilbert slots

\section{RESULTS AND DISCUSSION}

Minkowiski fractal shape is applied to the classical square antenna to enhance its performance. Complementary Split Ring Resonator (CSRR) has been used as slot on the antenna patch to reduce its size. In this work, two shapes of Complementary Split Ring Resonators are proposed. The classical split ring and Hilbert shape ring, this is shown in Fig. 3. Complementary 
Split Ring Resonator models are placed in a waveguide to calculate its S-parameters. From these parameters, we can calculate relative permittivity, relative permeability and refractive index. Resonances of classical ring and Hilbert ring are shown in Fig. 4 and Fig. 5. Relative permittivity and permeability of the unit cells are shown in Fig. 6 and Fig. 7. Refractive index of these models is shown in Fig. 8 and Fig 9.

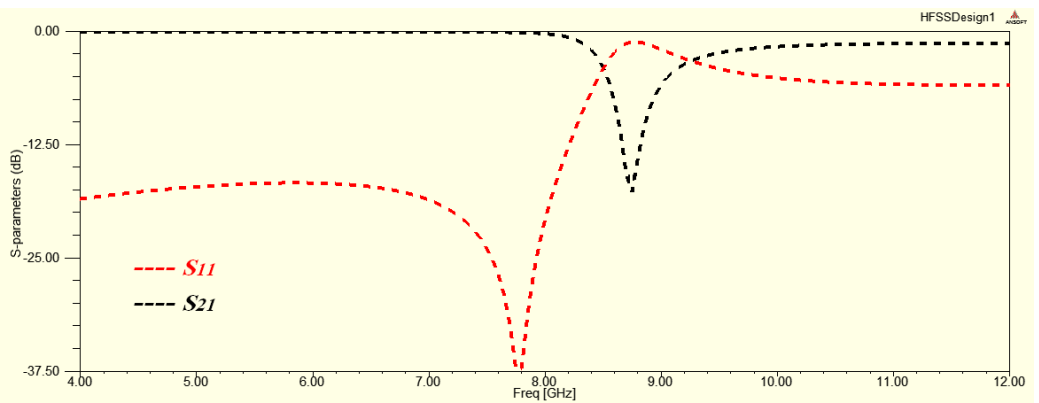

Fig. 4. S-parameters of classical split ring resonator

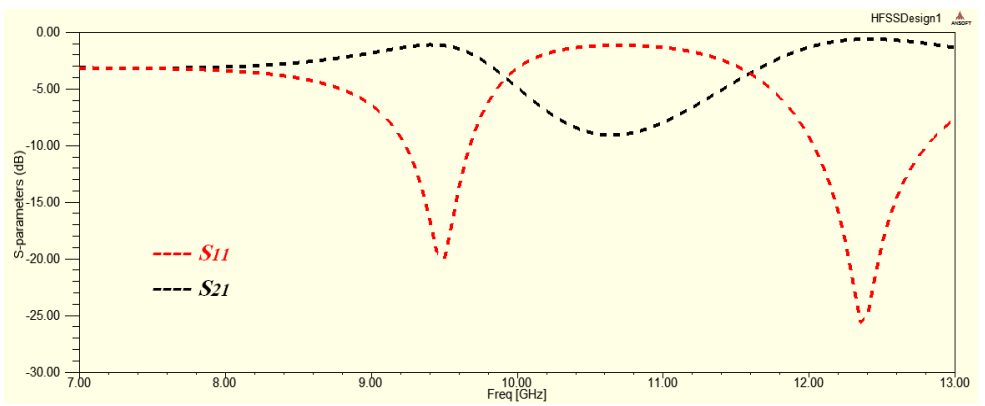

Fig. 5. S-parameters of Hilbert ring

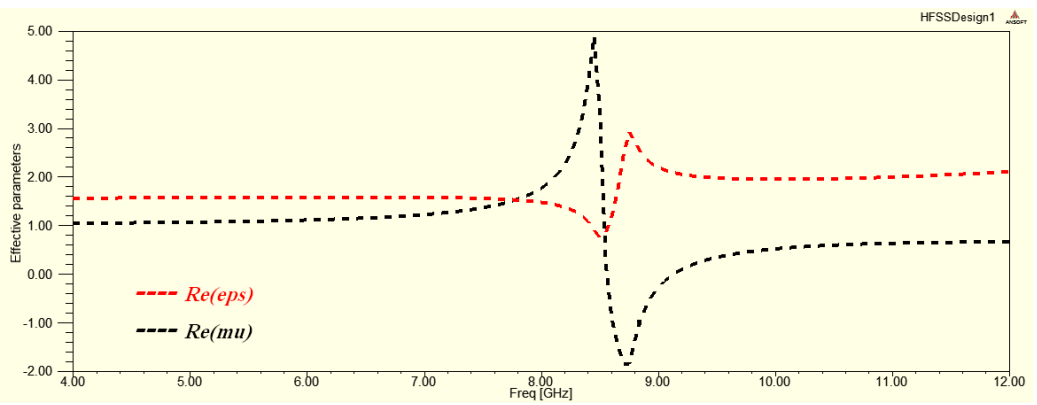

Fig. 6. Relative permittivity and permeability of classical split ring resonator

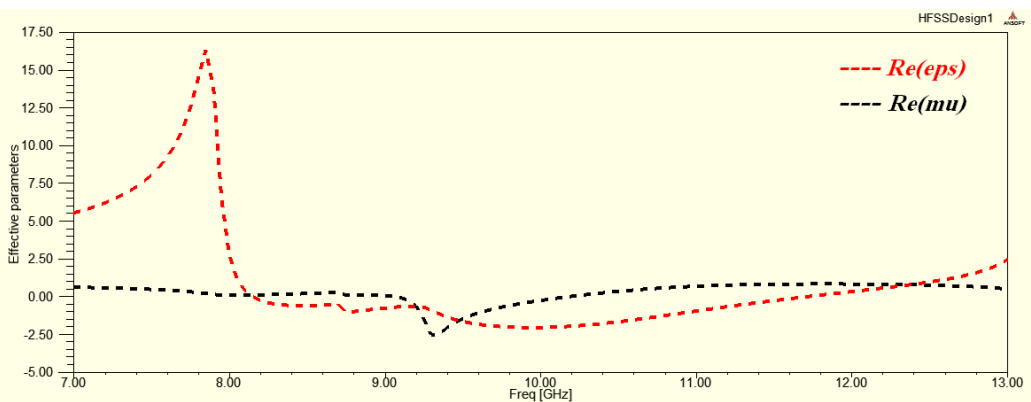

Fig. 7. Relative permittivity and permeability of Hilbert ring 


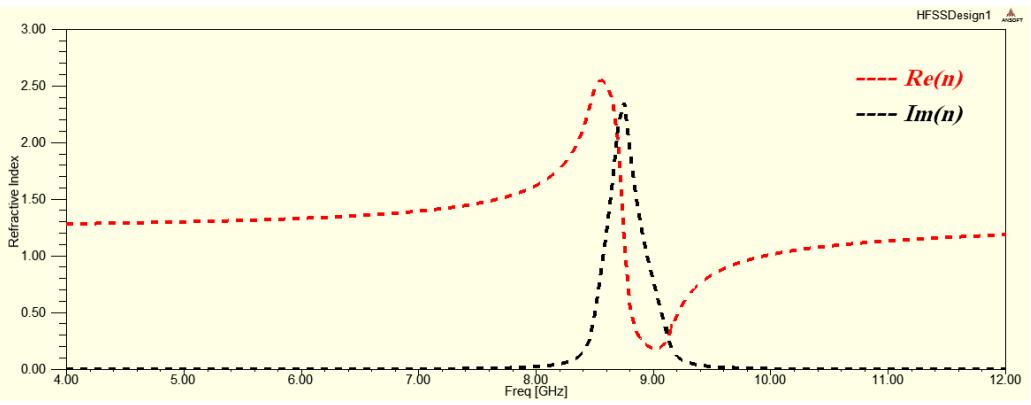

Fig. 8. Refractive index of classical split ring resonator

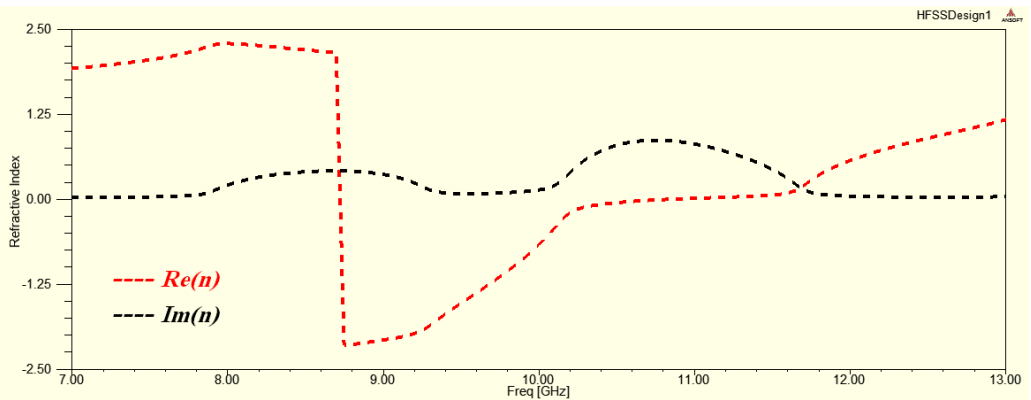

Fig. 9. Refractive index of Hilbert ring

Fractal Minkowski antenna has three resonance frequencies located at $4.5 \mathrm{GHz}, 8.9 \mathrm{GHz}$, and11.7 $\mathrm{GHz}$ as shown in Fig 10. Side length of the antenna is chosen to be $17 \mathrm{~mm}$ and the indentation width equals to $2 \mathrm{~mm}$. Applying classical ring and Hilbert ring on the patch as slots, will decrease the resonance frequencies. The new resonance frequencies after applying slots are shown in Fig. (11-12).

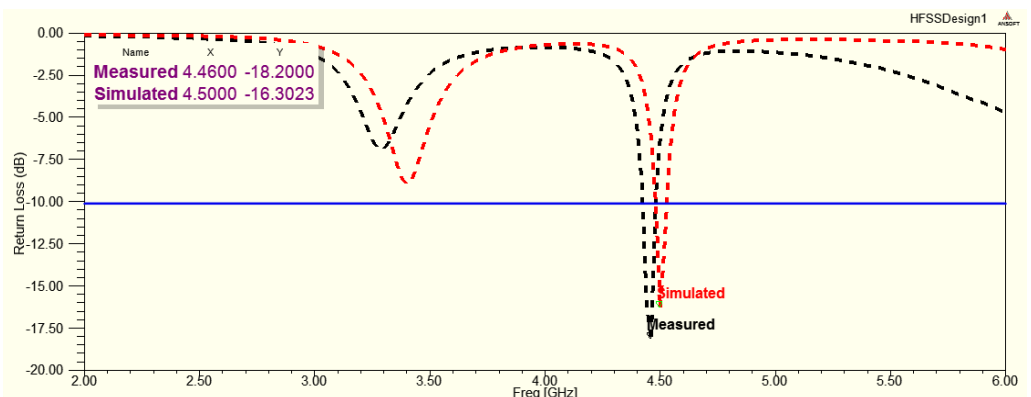

(a)

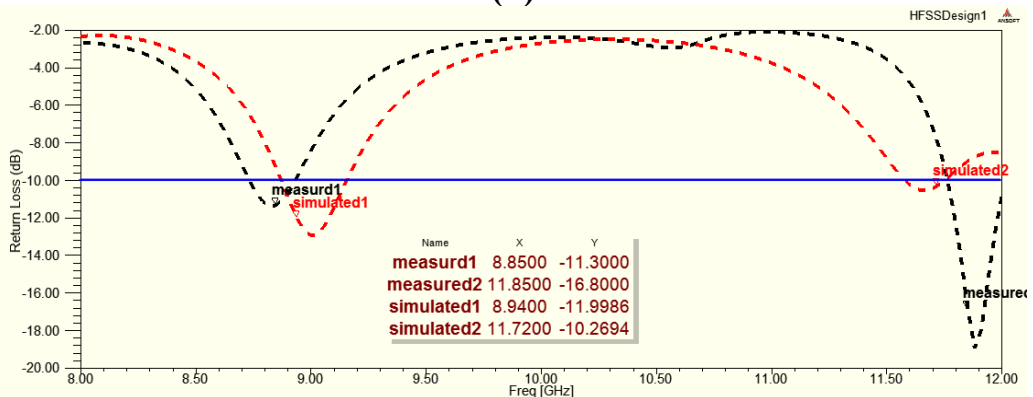

(b)

Fig. 10. S-parameters of Minkowiski Island antenna (a) $1^{\text {st }}$ resonance (b) $2^{\text {nd }}$ and $3^{\text {rd }}$ resonances 


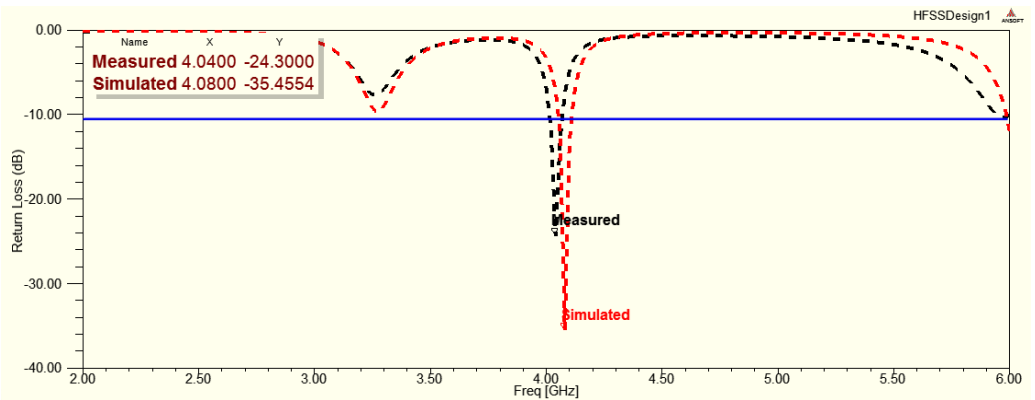

(a)

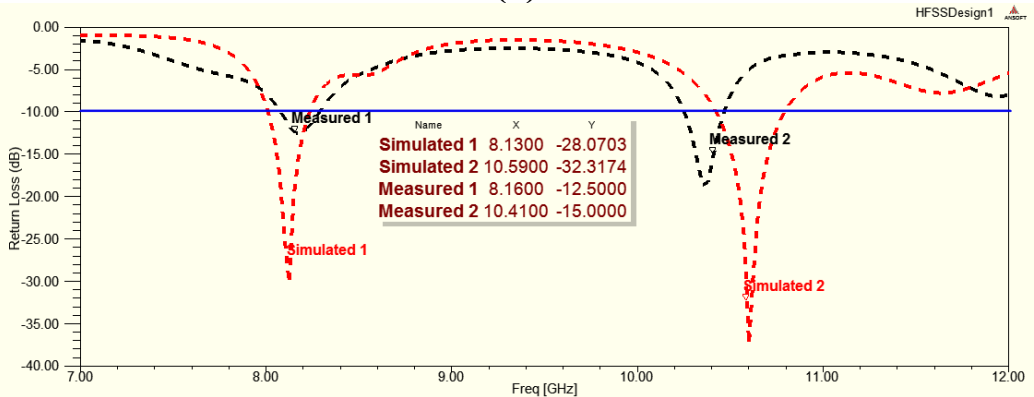

(b)

Fig. 11. S-parameters of Minkowiski Island antenna loaded with classical ring slot (a) $1^{\text {st }}$ resonance (b) $2^{\text {nd }}$ and $3^{\text {rd }}$ resonances

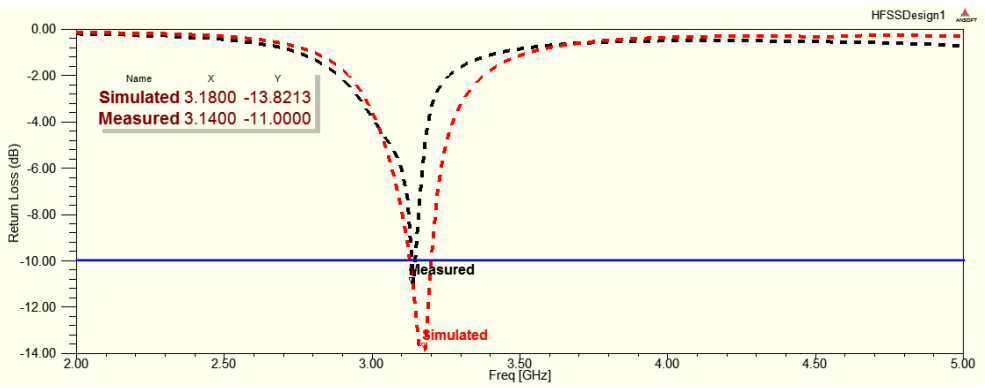

(a)

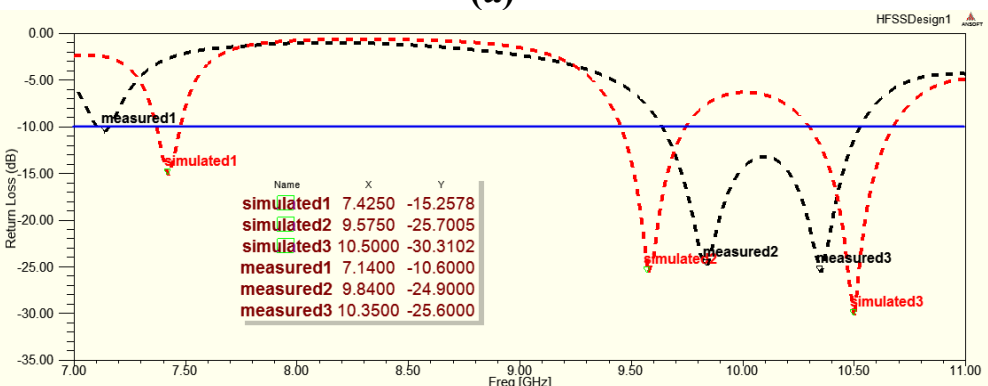

(b)

Fig. 12. S-parameters of Minkowiski Island antenna loaded with Hilbert ring slot (a) $1^{\text {st }}$ resonance (b) $2^{\text {nd }}$ and $3^{\text {rd }}$ resonances

This model is also fabricated and measured using vector network analyzer. Photograph of fabricated models is shown in Fig. 13. Measured S-parameters are shown in Fig. 14. 


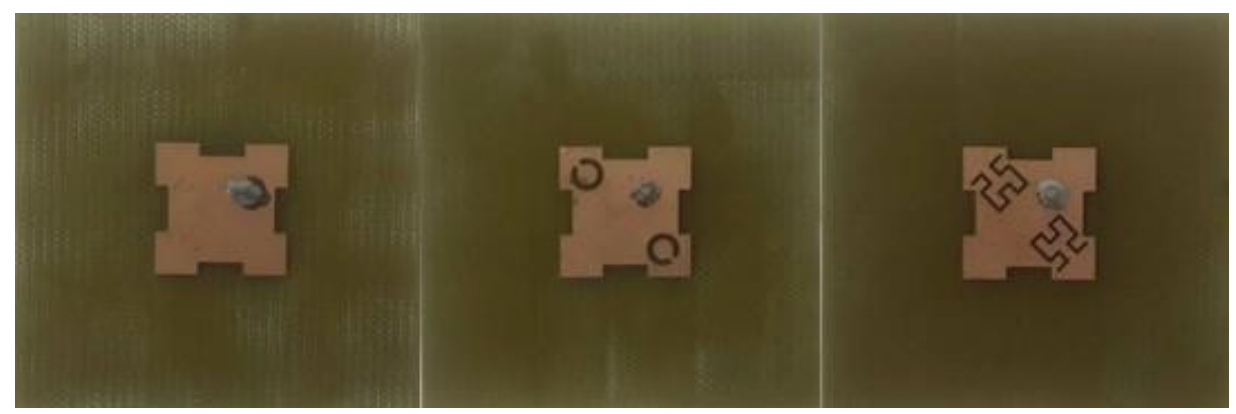

Fig. 13. Photo picture of fabricated antenna models

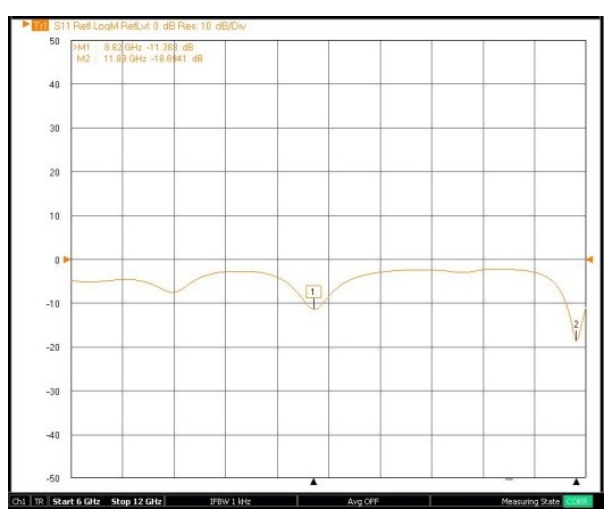

(a)

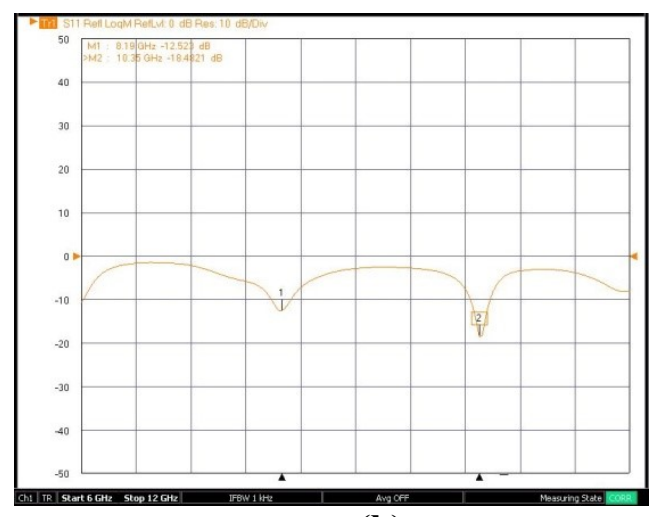

(b)

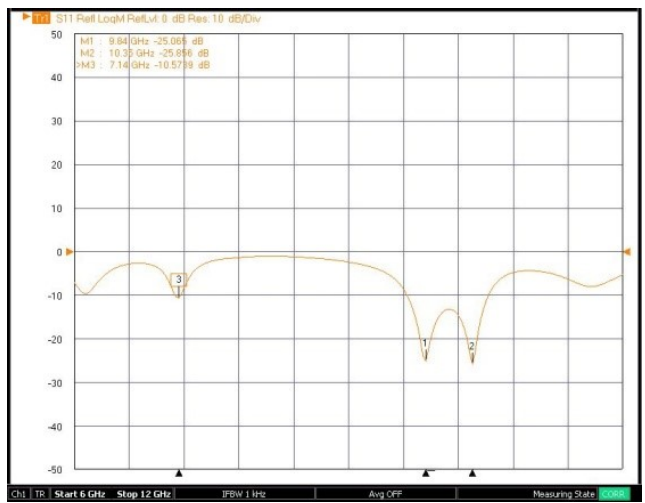

(c)

Fig. 14. Measured S-parameters for $2^{\text {nd }}$ and $3^{\text {rd }}$ frequency (a) Minkowski (b) Minkowski loaded with classical ring slot (c) Minkowski loaded with Hilbert ring

From the above figures of S-parameters, we notice a decrease in resonance frequencies of Minkowski antenna when slots are loaded on the patch. Decrease of resonance frequency that means a reduction of antenna area is obtained. Reduction of area when classical slot is loaded is equal to $18 \%$ of the Minkowski area. When Hilbert ring is slotted on the patch, we have gain a reduction of area equal to $33 \%$ for the third resonance frequency. If the antenna is used in application operate at the first resonance frequency, the reduction of area is equal to $50 \%$. The difference of reduction of antenna area among frequency bands is refers to the fractal shape of Hilbert ring.

The classical ring is a curved segment. If we neglect its width, it has dimension equal one. Hilbert ring has fractal dimension equals 1.26. The fractal dimension that is more than classical ring dimension explains high reduction of area when Hilbert ring is loaded on the patch.

Radiation pattern of Minkowski antenna models is shown in Figs. (14-16). 

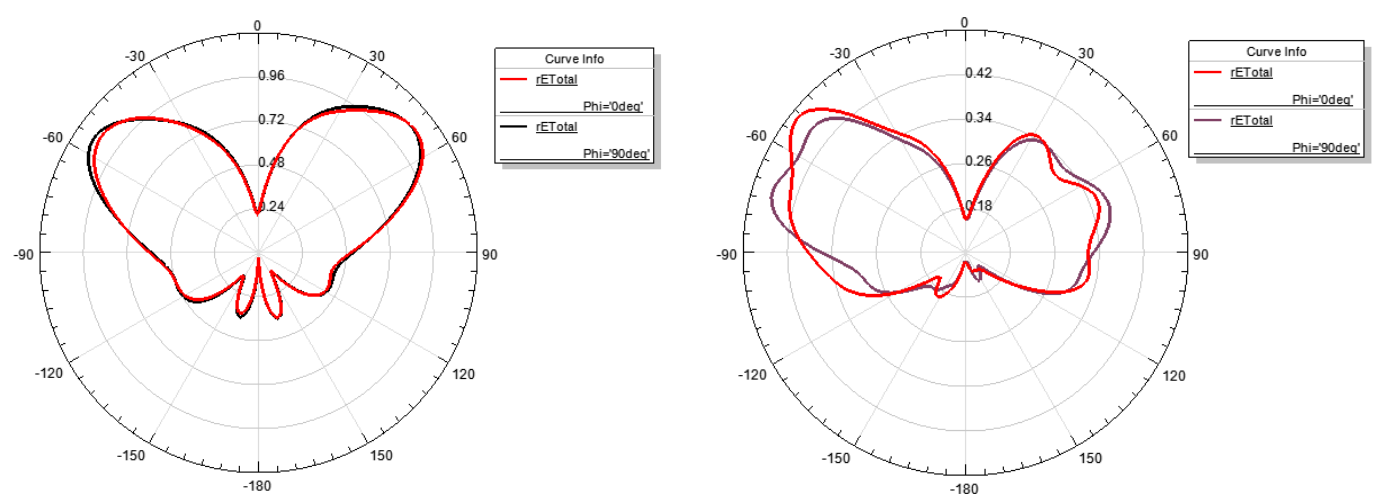

Fig. 14. Radiation pattern of Minkowski antenna at second and third frequency
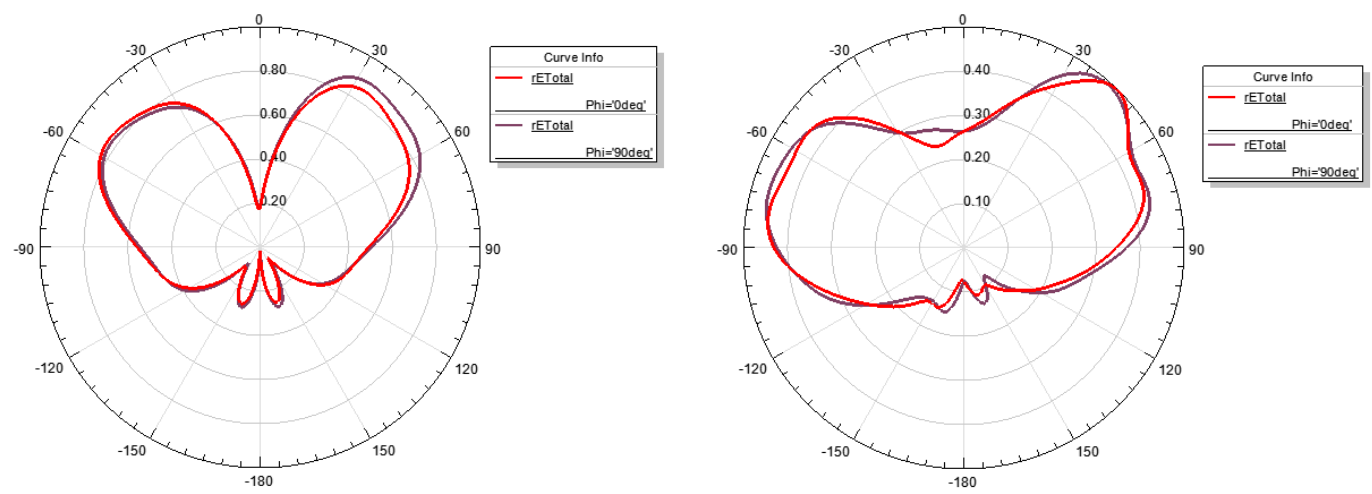

Fig. 15. Radiation pattern of Minkowski antenna loaded with classical ring slots at second and third frequency
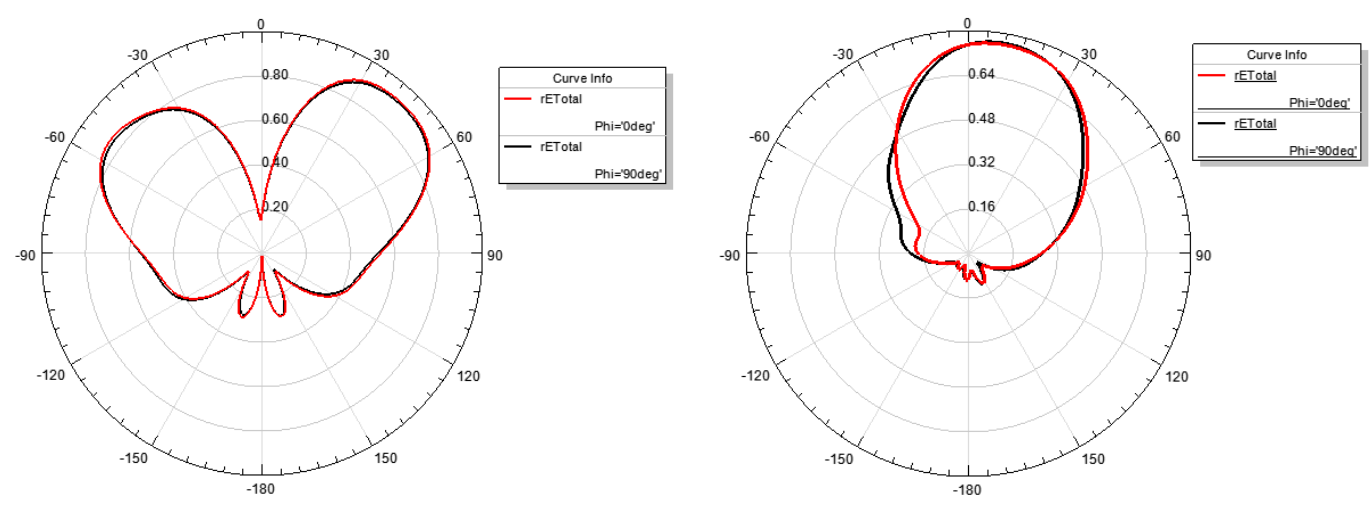

Fig. 16. Radiation pattern of Minkowski antenna loaded with Hilbert ring slots at second and third frequency

\section{CONCLUSION}

Antenna size reduction is of great interest in wireless communication systems. Fractal shapes can reduce the classical antenna size. Applying CSRR as a slot on the patch can make reduction in antenna size. First iteration of Minkowski Island is proposed. Classical split ring resonator and Hilbert ring are slotted on the antenna patch. Calculations made using (HFSS) software code. Simulation results show a reduction in the size of the antenna equals to $18 \%$ for model loaded with classical ring. Reduction equals to $50 \%$ relative to the first frequency and $33 \%$ relative to the third frequency for model loaded with Hilbert ring. Reduction ability is related to the fractal dimension. The fractal dimension of Hilbert ring equals to 1.26 while the dimension of classical ring equals to one. Thus, more reduction of antenna is obtained when Hilbert ring is used as complementary ring resonator. These models are fabricated and measured using vector network analyzer. Good agreement obtained of measured and simulated results. 


\section{Acknowledgment}

Author would like to express his thanks to the staff of Electronics Design Center, especially to Ghaleb N. Najm and Mahmood R. Muhsen from Ministry of Science and Technology, Iraq, for their support in the production of the antennas prototype.

\section{References}

[1] Veselago V., et al. "Negative Refractive Index Materials,"Journal of Computational and Theoretical Nanoscience, Vol.3, 2006,pp.1-30.

[2] Veselago V. "Electrodynamics of substrates with simultaneously negative values of $\varepsilon$ and $\mu$,'Sov. Phys. Usp., Vol. 10, No. 4, 1968, pp.509-514.

[3] Balmaz P., and Martin O. "Electromagnetic resonances in individual and coupled split-ring resonators," JOURNAL OF APPLIED PHYSICS , V. 92, no. 5, pp. 2929-2936

[4] Wu B., Li B., Su T., and Liang C. “Study on Transmission Characteristic of Split-ring Resonator Defected Ground Structure, ” PIERS ONLINE, Vol. 2, no. 6, 2006, pp. 710-714.

[5] Garg B., Singhal P.“Improving Principle Design of Rectangular SRR based Metamaterial Structure with Negative $\mu$ and $\varepsilon$ for Characteristics of Rectangular Microstrip Patch Antenna, "International Journal of Engineering Research, Vol.1, no.2, pp. 38-44

[6] Sarkar D., Saurav K., and Vaibhav K. "Design of a Novel Dual-band Microstrip Patch Antenna for WLAN/WiMAX Applications Using Complementary Split Ring Resonators and Partially Defected Ground Structure, "Progress In Electromagnetics Research Symposium proceedings, Taipei, March 25-28, 2013

[7] Bazrkar A., Gudarzi A., andMahzoonM."Miniaturization of rectangular patch antenna partially loaded with U-negative metamaterials,"International conference on Electronics, Biomedical and Its Applications (ICEBEA), Dubai, 2012, pp 289-292.

[8] Ye J., Cao Q., Tam W."Design and analysis of a miniature metamaterial microstrip patch antenna,” IEEE Antrnna Technology (iWAT), Hong Kong, 2011, pp 290-293.

[9] Huang J.“A review of antenna miniaturization techniques for wireless applications,” Jet Propulsion Laboratory, California Institute of Technology, 2001

[10] Waterhouse R., Targonski S., Kokotoff M."Design and performance of small printed antennas," IEEE trans. Antennas, 1998.

[11] Trippe A., Bhattacharya S., Papapolymerou J."Compact microstrip antennas on a high relative dielectric constant substrate at $60 \mathrm{GHz}$,'IEEE Antennas and Propagation (APSURSI), Spokane, 2011, pp 519-520.

[12] Ali J., Alsaedi H., Mohammed F., and Hammas H."APeano Fractal-based Dual-mode MicrostripBandpass Filters for Wireless Communication Systems" PIERS Proceedings, Moscow, Russia, , August 19-23, 2012, pp 888-892.

[13] DipaliSoren, RowdraGhatak, Rabindra Kishore Mishra, DipakRanjanPoddar, “ Wideband Sierpinski Carpet Fractal Shaped Cylindrical Dielectric Resonator Antenna for X-Band Application," Journal of Electromagnetic Analysis and Applications, 4, 2012, pp 9-14

[14] Pendry J., Holden A., Robbins D., and Stewart W."Magnetism from conductors and enhanced nonlinear phenom-ena, " IEEE Trans. on MTT, Vol. 47, 1999, 2075-2084.

[15] Smith D., Padilla W., Wier D., Nemat-Nasser, and Schultz S."Composite medium with simultaneously negative permeability and permittivity," Phys. Rev. Lett., Vol. 84, 2000, pp4184-4187. 
[16] Lee Y., Hao Y."Characterization of microstrip patch antennas on metamaterial substrates loaded with complementary split-ring resonators," Microwave And Optical Technology Letters, vol. 50, No. 8,August 2008, pp 2131-2135.

[17] Prombutr N., Akkaraaektharin P. "Analysis and Design Hilbert Curve Fractal Antenna Feed with Co-planar Waveguide for multi-band wireless communications, " International Journal of Engineering, Volume 2 Issue 3, 2007

[18] Kutter R. "Fractal Antenna Design,” B.Sc. Honor Thesis, BEE, University of Dayton, 1996.

[19] Gianvittorio J. "Fractal Antennas: Design, Characterization and Applications, "M.Sc. thesis, University of California, Los Angeles, 2000. 